\title{
Morphology Sponsor Defined Identifier
}

National Cancer Institute

\section{Source}

National Cancer Institute. Morphology Sponsor Defined Identifier. NCI Thesaurus. Code C117604.

One or more sponsor defined characters used to identify, name, or characterize the morphology finding. 